\title{
Influencia del proceso educativo en la consulta de ERCA sobre la elección de tratamiento renal sustitutivo
}

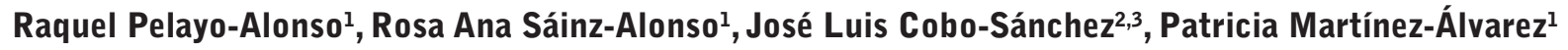 \\ ${ }^{1}$ Servicio de Nefrología. Hospital Universitario Marqués de Valdecilla. Santander. España \\ ${ }^{2}$ Subdirección de Cuidados. Servicio Cántabro de Salud. Santander. España \\ ${ }^{3}$ Escuela Universitaria de Enfermería Clínica Mompía. Universidad Católica de Ávila. Mompía. España
}

Como citar este artículo:

Pelayo-Alonso R, Sáinz-Alonso RA, Cobo-Sánchez JL, Martínez-Álvarez P. Influencia del proceso educativo en la consulta de ERCA sobre la elección de tratamiento renal sustitutivo.

Enferm Nefrol. 2020 Jul-Sep;23(3):267-272

\section{Resumen}

Introducción: La finalidad de las consultas de Enfermedad Renal Crónica es potenciar los autocuidados y autonomía del paciente. El proceso de información y elección de tratamiento renal sustitutivo en estos pacientes es un aspecto clave para su tratamiento.

Objetivo: Determinar si el tipo de proceso educativo que recibe el paciente en la consulta ERCA influye sobre la opción de tratamiento renal sustitutivo elegida.

Material y Método: Estudio observacional retrospectivo en pacientes seguidos en consulta de Enfermedad Renal Crónica en el periodo 2015-2020. Se recogieron datos clínicos y sociodemográficos de la historia clínica del paciente, opción terapéutica elegida por el paciente y proceso educativo recibido (sólo nefrólogo o equipo multidisciplinar: nefrólogo y enfermera).

Resultados: Se incluyeron 294 pacientes candidatos a tratamiento renal sustitutivo. El $51 \%$ de la muestra optó por hemodiálisis en centro como la opción más frecuente. Los pacientes que fueron educados por el equipo multidisciplinar presentan tasas de elección de hemodiálisis de $36,4 \%$ y de tratamiento conservador

\section{Correspondencia:}

Raquel Pelayo Alonso

Email: raquel.pelayo@scsalud.es del $18,2 \%$ frente al $56,7 \%$ y $5,1 \%$ respectivamente, del grupo informado sólo por el nefrólogo $(p<0,01)$.

Conclusiones: La hemodiálisis en centro es la opción más frecuente entre los pacientes. Cuando el paciente recibe un proceso informativo estructurado, sistemático y multidisciplinar la elección de las diferentes opciones tiende a equilibrarse y el tratamiento conservador es considerado una opción alternativa.

PALABRAS CLAVE: enfermedad renal crónica avanzada; educación sanitaria; toma de decisiones; terapia de reemplazo renal.

\section{Influence of the educational process in the ACKD consultation on the choice of renal replacement therapy}

\section{Abstract}

Introduction: The purpose of the Chronic Kidney Disease consultations is to enhance the patient's selfcare and autonomy. The process of information and choice of renal replacement therapy in these patients is a key aspect for their treatment.

Objective: To determine if the type of educational process that the patient receives in ACKD consultation influences the choice of the type of renal replacement treatment. 
Material and Method: Retrospective observational study in patients followed up in ACKD consultation in the period 2015-2020. Clinical and sociodemographic data were collected from the patient's medical history as well as the therapeutic option chosen by the patient and the educational process received (only nephrologist or multidisciplinary team: nephrologist and nurse).

Results: 294 patients who were candidates for renal replacement therapy were included. $51 \%$ of the sample chose in-centre haemodialysis as the most frequent option. The patients who were educated by a multidisciplinary team chose haemodialysis in $36.4 \%$ and conservative treatment in $18.2 \%$ versus $56.7 \%$ and $5.1 \%$ respectively, for the group that was informed by only the nephrologist $(p<0.01)$.

Conclusions: In-centre haemodialysis is the most frequent option among patients. When the patient receives a structured, systematic and multidisciplinary information process, the choice between the different options tends to balance out and conservative treatment is considered an alternative option.

KEYWORDS: advanced chronic kidney disease; health education; Decision making; renal replacement therapy.

\section{Introducción}

La Enfermedad Renal Crónica Avanzada (ERCA) es un problema de salud pública que aumenta su prevalencia de forma progresiva a medida que se produce un mayor envejecimiento de la población ${ }^{1}$.

Supone un importante problema de salud pública no sólo por su prevalencia en aumento, sino también por las comorbilidades que el paciente renal presenta y por el elevado coste socio-económico que suponen las diferentes opciones de tratamiento ${ }^{2,3}$.

Tras la promulgación de la ley 41/2002, básica reguladora de la Autonomía del paciente ${ }^{4}$, los pacientes tienen regulado su derecho a decidir libre y voluntariamente sobre las distintas opciones terapéuticas tras recibir una información apropiada, no sesgada e individualizada sobre todo su proceso asistencial ${ }^{1,5}$. Desde la Consulta de Enfermedad Renal Crónica Avanzada (CERCA) se atiende a pacientes con ERCA es estadio 4-5, es decir, con una tasa de filtrado glomerular $<30 \mathrm{ml} / \mathrm{min} / 1,73 \mathrm{~m}^{2}$. La finalidad de estas consultas es potenciar los autocui- dados y la autonomía del paciente ${ }^{6,7}$, implicando tanto al paciente como a su entorno más cercano en el proceso asistencial, con el objetivo de mejorar el estado de salud de los pacientes, retrasando el inicio del tratamiento renal sustitutivo (TRS) y que cuando éste llegue, los resultados de la elección sean los mejores para el paciente y su entorno ${ }^{8}$. Por lo tanto, el proceso de información y elección de TRS en el paciente ERCA es una etapa clave para su tratamiento ${ }^{5,8}$. Desde las CERCA se busca llevar a cabo un proceso educativo que fomente la toma de decisiones por parte del paciente, garantizando el respeto de sus valores, preferencias y estilo de vida9-11.

Sin embargo, muchos pacientes no reciben educación previa al inicio del TRS y cuando lo hacen, la información que reciben no es suficiente para comprender las diferentes alternativas terapéuticas ${ }^{11}$, lo que se traduce en que la hemodiálisis (HD) en centro siga siendo la opción terapéutica más frecuente para el inicio del TRS ${ }^{13}$.

Diversos estudios demuestran que cuando un paciente recibe educación sanitaria reglada y estructurada, el porcentaje de elección de terapias domiciliarias aumen$\operatorname{ta}^{1,2,5,7,14}$.

\section{Objetivo}

Determinar si el tipo de proceso educativo que recibe el paciente en la consulta ERCA influye sobre la opción de tratamiento renal sustitutivo elegida.

\section{Material y Método}

Se realizó un estudio observacional retrospectivo comparativo en pacientes seguidos en la consulta ERCA del Servicio de Nefrología del Hospital Universitario Marqués de Valdecilla (de referencia para la Comunidad Autónoma de Cantabria) entre el 1 de febrero de 2015 y el 31 de enero de 2020. Se excluyeron a los pacientes que no eran candidatos a TRS según sus características clínicas (enfermedad grave que condiciona la supervivencia corto plazo y que no es corregible mediante la diálisis o el trasplante renal, deterioro funcional y deterioro cognitivo o enfermedad psiquiátrica grave) y a aquellos pacientes que aún no habían elegido opción terapéutica.

Se recogieron datos clínicos y sociodemográficos de la historia clínica electrónica del paciente incluyendo sexo, edad, etiología de la enfermedad renal de base 
(según código ERA/EDTA), antecedentes personales de diabetes mellitus, grado de comorbilidad según el índice de Charlson (sin corrección para la edad), tasa de filtrado glomerular (según la ecuación CKD-EPI) en el momento en que se plantea la necesidad de TRS, opción terapéutica escogida por el paciente y el proceso educativo recibido.

En el periodo de estudio se establecen 2 fases, lo que diferencia el proceso educativo que recibe el paciente:

- Una primera fase, desde el 1 de febrero de 2015 hasta el 31 de enero de 2019, en la que el proceso educativo es proporcionado sólo por el nefrólogo.

- Una segunda fase, desde el 1 de febrero de 2019 hasta el 31 de enero de 2020, en la que entra en funcionamiento un equipo multidisciplinar, lo que conlleva que el proceso educativo sea llevado a cabo por la enfermera una vez que el paciente ha sido valorado por el nefrólogo.

Este proceso educativo multidisciplinar (nefrólogo y enfermera) es un proceso sistemático que consta de 4 fases secuenciales, y ha sido descrito previamente en otras publicaciones $\mathrm{s}^{3,9,10}$. En la primera fase se identifican las preferencias, valores y estilo de vida del paciente. Se continua con la fase de información, tanto al paciente como a su entorno, de las distintas opciones de TRS posibles. Esta fase se complementa con la entrega de información adicional, en soporte escrito o digital para completar la información que se proporciona al paciente. En la tercera fase, la enfermera se encarga de resolver aquellas dudas que el paciente plantea tras asimilar la información y deliberar con su entorno. EI proceso finaliza cuando el paciente se decide por una opción terapéutica.

A partir de las variables recogidas se elaboró una base de datos tipo Excel anonimizada. Para el análisis de los datos se utilizó el paquete estadístico PSPP v1.2. Se realizó un análisis descriptivo utilizando medidas de tendencia central para las variables cuantitativas; y frecuencias y porcentajes para las variables cualitativas. Se utilizaron los test $\mathrm{Chi}^{2}$, $\mathrm{t}$ de Student y contraste bilateral para la inferencia estadística, considerándose un valor $p<0,05$ como estadísticamente significativo.

El estudio se llevó a cabo cumpliendo la Ley orgánica $3 / 2018$, de 5 de diciembre de protección de datos personales y garantía de los derechos digitales.

\section{Resultados}

Durante el periodo de estudio, fueron seguidos en la consulta ERCA 586 pacientes, de los que se incluyeron en el estudio los 294 pacientes candidatos a TRS y que recibieron algún tipo de proceso educativo.

La edad media de la muestra fue $67,24 \pm 12,9$ años (mediana 69,5 años). El 70,75\% fueron hombres y la puntuación de comorbilidad media fue de 4,37 $\pm 1,96$ puntos (mediana 4 puntos). La tasa de filtrado glomerular en el momento en que se plantea la necesidad de TRS fue de $15 \pm 4,7 \mathrm{ml} / \mathrm{min} / 1,73 \mathrm{~m}^{2}$. Las características clínicas de los pacientes estudiados se detallan en la Tabla 1.

Tabla 1. Características clínicas de los pacientes candidatos a Tratamiento Renal Sustitutivo.

\begin{tabular}{lcc}
\hline & n & $\%$ \\
\hline $\begin{array}{l}\text { Sexo } \\
\text { - Hombre }\end{array}$ & 208 & $70,75 \%$ \\
- Mujer & 86 & $29,25 \%$ \\
\hline DM & & \\
- Si & 146 & $49,66 \%$ \\
- No & 148 & $50,34 \%$ \\
Nefropatía de base & & \\
- Glomerular & 52 & $17,69 \%$ \\
- Intersticial & 91 & $30,96 \%$ \\
- Sistémica & 73 & $24,83 \%$ \\
- Congénita & 26 & $8,84 \%$ \\
- Otras & 26 & $8,84 \%$ \\
- Desconocida & 26 & $8,84 \%$ \\
Opción TRS elegida por paciente & & \\
- Trasplante & 59 & $20,07 \%$ \\
- Diálisis Peritoneal & 59 & $20,07 \%$ \\
- Hemodiálisis & 151 & $51,36 \%$ \\
- Tratamiento conservador & 25 & $8,50 \%$ \\
\hline
\end{tabular}

DM: Diabetes Mellitus; TRS: tratamiento renal sustitutivo.

En la Tabla 2 se muestran las características clínicas de los pacientes según el profesional que lleva a cabo el proceso educativo (sólo nefrólogo o nefrólogo junto a enfermera).

La HD fue la opción de TRS escogida con mayor frecuencia durante el periodo de estudio, siendo la opción seleccionada por el $51 \%$ de los pacientes.

El grupo de pacientes que recibió el proceso educativo sistemático a cargo del equipo multidisciplinar (nefrólogo y enfermera) presenta tasas de elección de HD de 
Tabla 2. Características clínicas de los pacientes según el profesional que lleva a cabo el proceso educativo.

\begin{tabular}{|c|c|c|c|c|c|}
\hline & \multicolumn{2}{|c|}{ Nefrólogo (n 217) } & \multicolumn{3}{|c|}{ Nefrólogo y Enfermera (n 77) } \\
\hline & Media & DE & Media & DE & $\mathbf{p}$ \\
\hline Edad & 66,78 & 12,45 & 68,54 & 14,17 & NS \\
\hline IC Charlson (puntos) & 4,37 & 1,98 & 4,36 & 1,90 & NS \\
\hline \multirow[t]{2}{*}{$\mathrm{FG}\left(\mathrm{ml} / \mathrm{min} / 1,73 \mathrm{~m}^{2}\right)$} & 15,18 & 4,99 & 14,52 & 3,71 & NS \\
\hline & n & $\%$ & n & $\%$ & p \\
\hline \multicolumn{6}{|l|}{ Sexo } \\
\hline - Hombre & 156 & $71,89 \%$ & 52 & $67.5 \%$ & NS \\
\hline - Mujer & 71 & $28,11 \%$ & 25 & $32,5 \%$ & \\
\hline \multicolumn{6}{|l|}{ DM } \\
\hline - $\mathrm{Si}$ & 108 & $49,8 \%$ & 38 & $49,35 \%$ & NS \\
\hline - No & 109 & $502 \%$ & 39 & $50,65 \%$ & \\
\hline \multicolumn{6}{|l|}{ Nefropatía base } \\
\hline - Glomerular & 34 & $15,7 \%$ & 18 & $23,4 \%$ & NS \\
\hline - Intersticial & 74 & $34,1 \%$ & 17 & $22,1 \%$ & \\
\hline - Sistémica & 48 & $22,1 \%$ & 25 & $32,5 \%$ & \\
\hline - Congénita & 21 & $9,7 \%$ & 5 & $6,5 \%$ & \\
\hline - Otras & 21 & $9,7 \%$ & 5 & $6,5 \%$ & \\
\hline - Desconocida & 19 & $8,8 \%$ & 7 & $9,1 \%$ & \\
\hline
\end{tabular}

IC: Índice de Comorbilidad; FG: Filtrado Glomerular; DM: Diabetes Mellitus; NS: no significativo $(p>0,05)$

$36,4 \%$ y de tratamiento conservador del $18,2 \%$ frente al $56,7 \%$ y $5,1 \%$ respectivamente, del grupo informado sólo por el nefrólogo, siendo estadísticamente significativo $(p<0,01)$.

En la Figura 1 se muestra la distribución de las opciones terapéuticas elegidas por el paciente según el tipo de proceso educativo y el profesional que lo lleva a cabo.

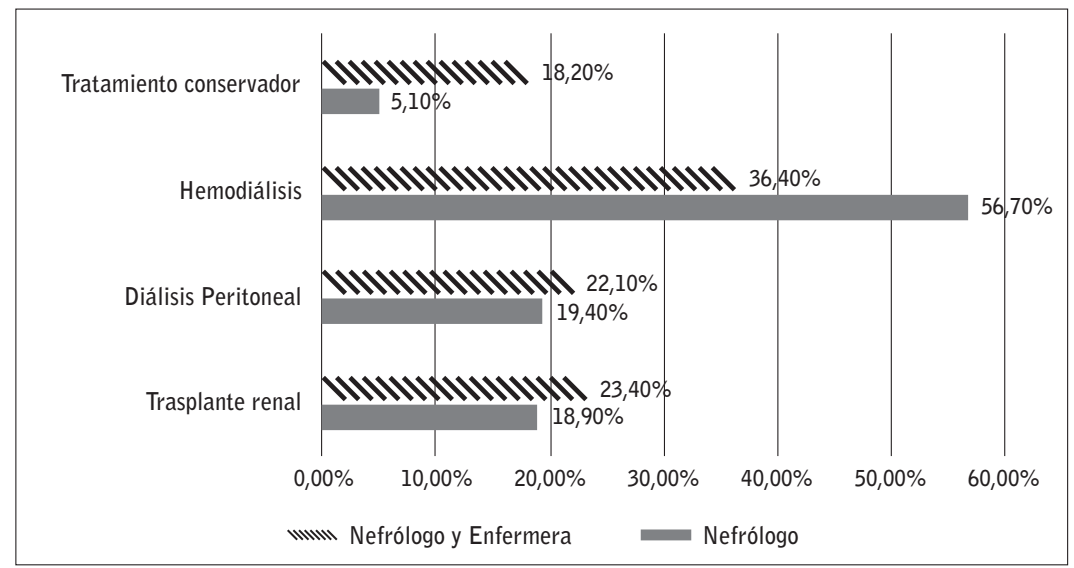

Figura 1. Distribución de la opción de tratamiento renal sustitutivo elegida por el paciente según el tipo de proceso educativo y el profesional que lo lleva a cabo.

\section{Discusión}

Tras la promulgación de la Ley 41/2002, es de obligado cumplimiento por el personal sanitario el fomentar el derecho a la información sanitaria ${ }^{4}$. El reconocimiento de la autonomía de paciente implica que las decisiones sanitarias ya no sólo dependen del criterio de los profesionales sanitarios de referencia, sino que es el propio paciente quién debe tomar la decisión sobre cómo quiere ser tratado ${ }^{1,8}$. Para ello es necesario proporcionar al paciente un proceso educativo sistemático que proporcione información no sesgada de las diferentes opciones terapéuticas, permitiendo al paciente identificar sus valores y preferencias, y tomar una decisión acorde con estos valores ${ }^{8,11}$.

La revisión sistemática de Van de Bosch y cols. ${ }^{12}$, pone de manifiesto la necesidad e importancia de que la educación previa al inicio del TRS sea llevado a cabo por un equipo multidisciplinar, que incluya, al menos un nefrólogo, una enfermera, un dietista y 
un trabajador social. Ludlow y cols. ${ }^{15}$ refieren que, pese a contar con un equipo multidisciplinar, una alta proporción de los pacientes son educados por la enfermera, ya que los nefrólogos tienen un tiempo limitado para llevar a cabo una educación personalizada. En nuestro medio, tras la incorporación de la figura de la enfermera a la Consulta ERCA, el proceso educativo personalizado es llevado a cabo por dicho profesional. Según nuestros datos, en aquellos pacientes en los que el proceso educativo es llevado a cabo por la enfermera, se observan cambios en la distribución de las diferentes opciones de TRS, con menor elección de la hemodiálisis en centro y mayor proporción de elección del tratamiento conservador. Es conocido que la educación previa a la diálisis aumenta la proporción de técnicas domiciliarias ${ }^{1,2,5,7,14}$. Sin embargo, los registros oficiales, no muestran una distribución equilibrada de las diferentes opciones del TRS. El informe de Diálisis y Trasplante del Registro Español de Enfermos Renales correspondiente al año $2018^{13}$, muestra que la HD sigue siendo la opción terapéutica más frecuente entre los pacientes incidentes, con un $78,9 \%$. En términos generales, nuestros datos también muestran una mayor elección de la HD en centro, aunque con un porcentaje del $51 \%$, datos similares a otras series 5 . Sin embargo, cuando el paciente es sometido a un proceso educativo sistemático y estructurado, a cargo del nefrólogo y la enfermera y no sólo por el nefrólogo, la elección de HD disminuye hasta el 36,4\% como refieren los datos. Es necesario incidir, que nuestros datos se refieren a la opción de TRS escogida, no a la técnica con la que el paciente inicia realmente su tratamiento, aspecto que no se ha valorado en este estudio pero que puede ser objetivo de futuras investigaciones.

Prieto y cols ${ }^{11}$ en su serie consiguen que la tasa de elección de HD y diálisis peritoneal (DP) tienda a igualarse al seguir este mismo proceso educativo estructurado. EI estudio ERCA ${ }^{3}$ también muestra una distribución similar a nuestros datos cuando el proceso educativo es aplicado, con una tasa de elección de DP del $26 \%$, valor muy superior al que muestran los registros oficiales ${ }^{13}$.

Cabe destacar el aumento significativo de la tasa de elección del tratamiento conservador en nuestra serie, cuando el paciente recibe el proceso educativo a cargo del equipo multidisciplinar. Las causas que motivan que el paciente no sea candidato a TRS están referidas en la bibliografía $^{14,16}$. Sin embargo, hay ocasiones en las que el paciente, pese a presentar características clínicas que hacen posible alguna de las opciones de TRS, escoge, por decisión propia, el tratamiento conservador. Teruel y cols. ${ }^{16}$ en su estudio, detallan un porcentaje de tratamiento conservador del $39 \%$ en los pacientes atendidos en su consulta
ERCA, siendo destacable que el $26 \%$ de los pacientes se mantiene en tratamiento conservador por decisión propia. Este porcentaje es similar a los datos que se obtienen en nuestro estudio cuando el paciente recibe un proceso educativo sistemático a cargo del equipo multidisciplinar. Cifras similares son alcanzadas también por Aguilera y cols. $^{10} 0$ el estudio ERCA ${ }^{3}$, con tasas del $18 \%$ y $25 \%$ respectivamente. Estos datos pueden indicar, que cuando el paciente recibe una información estructurada, sistemática y adaptada durante la fase terminal de su ERC, la opción de tratamiento conservador es contemplada por el paciente y su entorno.

Cuando el paciente recibe un proceso educativo adecuado, en el que se proporciona una información apropiada, material audiovisual complementario y se involucra tanto al paciente como a su entorno, la tasa de elección de las distintas modalidades de TRS tiende a equilibrarse.

Como limitaciones del estudio, indicar que nuestro estudio no es de tipo experimental, y que, por lo tanto, no se siguió una distribución aleatorizada de los pacientes que recibieron un proceso educativo u otro. Previo a la creación de la consulta de enfermería ERCA, todos los pacientes eran informados por el nefrólogo y tras la incorporación de la enfermera, todos los pacientes reciben un proceso educativo estructurado a cargo del nefrólogo y la enfermera. Además, debido a la reciente incorporación de la enfermera en la Unidad ERCA, la distribución de los pacientes en ambos periodos no es equitativa.

Como conclusión, podemos decir que, según nuestros datos, existe diferencia en cuanto a la opción de TRS elegida, en función de si el proceso educativo es estructurado, sistemático y multidisciplinar, aumentando en este último caso la opción de tratamiento conservador y disminuyendo la hemodiálisis en centro. Además, un proceso educativo correcto permite al paciente y a su entorno contemplar el tratamiento conservador como una alternativa más de tratamiento para la enfermedad renal crónica.

La creación de equipos multidisciplinares debe ser considerada para poder proporcionar un proceso educativo estructurado que facilite la libre elección del paciente, de forma autónoma y acorde a sus preferencias y valores.

Recibido: 20-08-2020

Revisado: 25-08-2020

Modificado: 27-08-2020

Aceptado: 28-08-2020 


\section{Bibliografía}

1. Navarro M, Navarro N. Importancia de la consulta enfermera como facilitador de la toma de decisiones en la consulta ERCA. ENE Revista de Enfermería 2013;7(3). [Consultado 3 may 2020]. Disponible en: http://ene-enfermeria.org/ojs/index.php/ENE/article/ view/333.

2. Wong $B$, Ravani $P$, Oliver MJ, Holroyd-Leduc J, Venturato $L$, Amit $X$ et al. Comparison of Patient Survival Between Hemodialysis and Peritoneal Dialysis Among Patients Eligible for Both Modalities. AJKD 2018;71(3):344-51.

3. Guerrero MA, Toro FJ, Batalha P, Salgueira M, González F, Marrero S y cols. Estudio ERCA. Características basales, evaluación de la aplicación de la información estructurada para la elección de tratamiento renal sustitutivo y evolución a un año de los pacientes incidentes en la consulta ERCA. Nefrología 2019;39(6):629-37.

4. Ley $41 / 2002$, de 14 de noviembre, básica reguladora de la autonomía del paciente y de derechos y obligaciones en materia de información y documentación clínica. B0E num. 274, de 15 de noviembre de 2002:40126-32.

5. Heredia J, Lorente C, Astorga F, Chica J. Factores diferenciadores en la elección de tratamiento sustitutivo renal en nuestra consulta de enfermedad renal crónica. Enferm Nefrol 2013;16(Supl 1):34-9.

6. García H, Rodríguez R, Celadilla 0, Bajo A, Sánchez $R$, Peso $G$ y cols. Abordaje interdisciplinar enfermera-psicólogo en la consulta de enfermedad renal crónica avanzada: objetivos y protocolo. Nefrología 2013;33(1):139-40.

7. Astudillo E, Rivas A, Sánchez E, Rodríguez M, Vidau P, Rodríguez MC, Díaz MC. Consulta de enfermedad renal crónica avanzada: ¿Influye la educación sanitaria sobre la modalidad de tratamiento elegido? Nefrología 2019;30(3):322-3.
8. Lorenzo M, Sánchez $B$, Cepeda I, Viera DM, Alonso I. La elección de TRS en la consulta ERCA: revisión de resultados y propuestas de mejora asistencial. Enferm Nefrol 2014;17(Supl 1):88-108.

9. Renau EM, García, Cerrillo V, Folch MJ, Bort J, Aircat CC. Medidas adoptadas para conseguir que todos los pacientes que inicien tratamiento pasen por la consulta ERCA de enfermería. Enferm Nefrol 2015;18 (Supl 1):93-100.

10. Aguilera AI, Alonso AC, Linares B, Prieto S, García $\mathrm{L}$, Prieto M. Valoración de la elección de tratamiento conservador en la Enfermedad Renal Crónica. Enferm Nefrol 2019;22(1):52-8.

11. Prieto-Velasco M, Quiros P, Remon C. Spanish Group for the Implementation of a Shared Decision Making Process for RRT Choice with Patient Decision Aid Tools (2015) The Concordance between Patients' Renal Replacement Therapy Choice and Definitive Modality: Is It a Utopia? PLoS ONE 10(10): e0138811. doi:10.1371/journal.pone.0138811.

12. Van den Bosch J, Simone Warren D, Rutherford P. Review of predialysis education programs: a need for standardization. Patient Preference and Adherence 2015;9:1279-91.

13. Informe de diálisis y trasplante 2018 del Registro Español de Enfermos Renales. [Consultado 15 feb 2019]. Disponible en: https://www.senefro.org/contents/webstructure/SEN_2019_REER_modificada.pdf.

14. Martínez Y, Toapanta NG, Nava N, Barbosa F, Montes $\mathrm{R}$, Guerrero MA. Supervivencia de pacientes de edad avanzada ( $\geq 70$ años) con enfermedad crónica estadios 4-5: diálisis vs tratamiento conservador. Nefrología 2016;36(3):283-91.

15. Ludlow MJ, George CR, Hawley CM et al. How australian nephrologists view home dialysis: results of a national survey. Nephrology (Carlton). 2011;16:446-52.

16. Teruel JL, Burguera V, Gomis A, Rivera M, Fernández $\mathrm{M}$, Rodríguez N, Quereda C. Elección de tratamiento conservador en la enfermedad renal crónica. Nefrología 2015;35(3):273-9.

Este artículo se distribuye bajo una Licencia Creative Commons Atribución-NoComercial 4.0 Internacional. https://creativecommons.org/licenses/by-nc/4.0/ 